\title{
Depression, Self-Criticism, and Nonsuicidal Self-Injury: A Prospective Investigation in Young Adults
}

\author{
Sarah E. Victor, Sean M. Mitchell, and Paul B. Ingram \\ Department of Psychological Sciences, Texas Tech University
}

\begin{abstract}
Author Note
Sarah E. Victor (iD https://orcid.org/0000-0002-7944-7299

Sean M. Mitchell (D) https://orcid.org/0000-0002-3729-7368

Paul B. Ingram (D) https://orcid.org/0000-0002-5409-4896
\end{abstract}

We have no conflicts of interest to disclose. Time for research was supported, in part, by a grant from the National Institute of Mental Health (L30 MH120575, PI: Sean M. Mitchell). Paul B. Ingram receives research funding from the University of Minnesota Press, publisher of the MMPI family of instruments. Study procedures were approved by the Texas Tech University Institutional Review Board (IRB\#2019-625).

Correspondence concerning this article should be addressed to Sarah E. Victor, Department of Psychological Sciences, Texas Tech University, Box 42051, Lubbock, TX 794092051. Email: Sarah.Victor@ttu.edu

Draft version 1.0, 8/3/2021. This paper has not yet been peer reviewed. Please do not reproduce without authors' permission. 


\begin{abstract}
Nonsuicidal self-injury (NSSI) is associated with significant distress, impairment, and suicide risk among young adults. Negative affectivity and self-critical cognitions are linked to NSSI but have rarely been considered jointly alongside indicators of NSSI severity (e.g., frequency, recency, number of methods). To address this gap, we examined depressive symptoms and self-criticism as concurrent and prospective predictors of NSSI urges among depressed young adults over six weeks. Baseline NSSI severity was positively related to baseline depressive symptoms, which predicted follow-up NSSI urges; however, baseline depressive symptoms did not predict later NSSI urges when controlling for baseline NSSI severity. In contrast, self-criticism was not associated with baseline NSSI severity, but predicted follow-up NSSI urges, even when controlling for prior NSSI severity. Using within-person longitudinal path models, depressive symptoms were positively associated with NSSI urges at the same assessment but did not prospectively predict later NSSI urges; however, self-criticism did predict later NSSI urges, but was not related to concurrent NSSI urges. Thus, depressive symptoms may serve as an indicator of overall NSSI risk but may be less informative than self-criticism in predicting within-person changes in NSSI urges over time (e.g., during treatment). These findings inform NSSI theories and highlight potential NSSI prevention and intervention targets.
\end{abstract}

Keywords: nonsuicidal self-injury; self-criticism; depression; longitudinal 


\section{Depression, Self-Criticism, and Nonsuicidal Self-Injury: A Prospective Investigation in Young Adults}

Nonsuicidal self-injury (NSSI) is the deliberate, self-inflicted damage to bodily tissues without suicidal intent and for purposes that are not socially sanctioned (International Society for the Study of Self-Injury, 2018). NSSI often begins in adolescence or young adulthood (Klonsky, 2011), and is highly prevalent among college students (e.g., approximately $10.3 \%$ during their first year; Kiekens et al., 2019). NSSI is associated with psychosocial difficulties, such as low social support (Trepal et al., 2015), emotion dysregulation (Wolff et al., 2019), and stigma experienced towards oneself and from others (Burke et al., 2019). However, NSSI is often experienced as reinforcing (Chapman et al., 2006; Hooley \& Franklin, 2018), and greater engagement with and severity of NSSI longitudinally predicts subsequent NSSI over time (Franklin et al., 2014; Prinstein et al., 2010; Wilkinson et al., 2011). Of great concern, individuals who engage in NSSI are at markedly elevated risk for suicidal thoughts and behaviors, including suicide attempts and death by suicide (Ribeiro et al., 2016). Thus, research is needed to clarify risk factors for urges to engage in NSSI, such as depression symptoms, selfcriticism, and characteristics of NSSI history.

NSSI has been associated with a variety of psychopathological experiences, including internalizing disorders and those with core diagnostic components which cover affective/emotional dysregulation (Bentley et al., 2015; Turner et al., 2018). NSSI is particularly common among individuals with mood disorders, such as depression and bipolar disorder. These diagnoses are characterized by intense, and sometimes volatile, negative affective experiences (Hepp et al., 2017), which often trigger NSSI (Kiekens et al., 2020; Victor et al., 2019). Both self-report (Claes et al., 2010) and ecological momentary assessment (Burke et al., 2021a) 
research has demonstrated that negative affective experiences central to these diagnoses prompt NSSI urges and behaviors, and that NSSI itself transiently decreases negative affect, further reinforcing self-harm behavior (Gordon et al., 2010). These relationships are consistent with theoretical models of NSSI onset and maintenance, including those that highlight the role of experiential avoidance (Chapman et al., 2006), pain-offset relief (Franklin et al., 2013), benefits versus barriers to NSSI (Hooley \& Franklin, 2018), and functional models of NSSI (Klonsky et al., 2015). The link between negative affective experiences (e.g., depressive symptoms) and NSSI broadly has been well established; however, additional research is needed to understand the link between depressive symptoms and urges to engage in NSSI over brief periods of time.

Negative affect regulation functions of NSSI are the most highly endorsed by those who engage in self-injury, but self-punishment motivations are also common reasons for NSSI (Taylor et al., 2018). Although psychopathology that co-occurs with NSSI typically includes negative affect as a core component, they are also often characterized by feelings of worthlessness, excessive guilt, poor self-image, and unstable identity (Gad et al., 2019; Zahn et al., 2015). Extant literature has demonstrated that self-relevant negative emotions, such as shame and self-disgust, are particularly connected to NSSI (Victor et al., 2014), highlighting the potentially important role of self-criticism as a risk factor for NSSI urges and behaviors (Zelkowitz \& Cole, 2019). Self-criticism has shown a prospective association with NSSI (Perkins et al., 2020), and some EMA work supports these associations in daily life (Burke et al., 2021b). Taken together, both depression symptoms and self-criticism may serve as important, clinically relevant predictors of NSSI urges.

Research focused on elucidating the possible mechanisms underlying NSSI has expanded over the past few decades but has been limited by a focus on primarily cross-sectional, 
correlational methods to examine associations between affective and cognitive experiences and NSSI engagement. These methodologies are unable to clarify which NSSI correlates may serve as consequences, versus causes, of NSSI, due to the inability to examine temporal associations between potential risk factors and NSSI itself. Further, much of this research examines lifetime engagement in NSSI as the outcome of interest, which precludes examination of factors associated with continued (versus ceased) NSSI engagement and may be subject to recall biases (Robinson \& Wilson, 2020). Even prospective work has typically considered changes in NSSI over months or years (Fox et al., 2015); while informative, this research often lacks the temporal resolution to examine the ordering of changes in NSSI risk factors between assessment windows. Therefore, further research is needed to examine risk factors for NSSI urges over shorter timeframes, which is likely more informative regarding real-world NSSI risk (Rodríguez-Blanco et al., 2018).

To meet this need in the literature, to better inform prevention and intervention of realworld NSSI, and to examine prospective predictors of NSSI urges among those at highest risk, we utilized a four-wave, six-week longitudinal design examining risk for NSSI urges among young adults with elevated depressive symptoms and a history of NSSI $(n=92)$. First, we examined how baseline NSSI characteristics known to be associated with clinical severity (recency, frequency, number of methods) contributed to NSSI urges reported over follow-up. We hypothesized that participants with more recent (1A), more frequent (1B), and more versatile (e.g., a greater number of methods; 1C) NSSI would exhibit elevated NSSI urges across the follow-up period. Second, we considered how baseline measures of depressive symptoms and self-criticism may relate to follow-up NSSI urges. We hypothesized that both baseline depressive symptoms (2A) and self-criticism (2B) would independently predict follow-up NSSI urges. We 
further hypothesized that these associations with depressive symptoms (2C) and self-criticism (2D) would no longer be significant after controlling for baseline characteristics of NSSI, which we expected to be correlated with baseline depressive symptoms and self-criticism. Finally, we investigated how depressive symptoms and self-criticism reported at each follow-up wave would contribute to concurrent and prospective (lagged) NSSI urges. We hypothesized that withinperson increases in both depressive symptoms (3A) and self-criticism (3B) over time would be associated with concurrently reported NSSI urges; we treated the examination of depressive symptoms and self-criticism as prospective predictors of NSSI urges at the next follow-up as exploratory, as prior research in this area is limited.

\section{Method}

\section{Participants and Procedures}

Participants were young adult college students recruited from a large, public university in the southwestern United States for a study on depression (demographic details below). The study was advertised using physical and virtual advertisements distributed across campus and via email to the entire student body on a weekly basis, and participants were paid with an Amazon gift card (rather than via course credit). Thus, the sample is likely more representative of the student body generally, relative to studies that recruit from Psychology Department subject pools. All study procedures were approved by the university's Institutional Review Board and conducted in accordance with American Psychological Association ethical standards.

Interested individuals $(N=327)$ completed an online screening for the study using the Patient Health Questionnaire 9-item version (PHQ-9; Kroenke \& Spitzer, 2002). Students who scored in the moderate depression range or higher (total score $\geq 10 ; n=290$ ) were invited to complete an in-person baseline assessment battery in a campus laboratory; a total of 152 students 
(52.4\% of qualifying interested individuals) completed the baseline assessment battery. The session involved completing various self-report questionnaires, as well as the Minnesota Multiphasic Personality Inventory-2-Restructured Form-Expanded (MMPI-2-RF-EX), an enhanced version of the MMPI-2-RF (Ben-Porath \& Tellegen, 2008/2011), from which the MMPI-2-RF may be scored.

Participants were sent a link via email and text message to access web-based questionnaires at two, four, and six weeks following the baseline session. The measures in these questionnaires were keyed to assess experiences, thoughts, and feelings over the preceding twoweek interval (see below). The survey was available for 24 hours.

Data were removed for participants with an invalid MMPI-2-RF profile $(n=13)$ and/or incorrect answers on more than one of five attention check items embedded within the Qualtrics questionnaire battery $(n=2)$ during their in-person session, leaving 137 individuals with valid baseline data. Of these, 123 (89.78\%) completed at least one follow-up wave, and 104 (75.91\%) reported a lifetime history of NSSI. The analytic sample for this manuscript is comprised of 92 individuals ( $67.15 \%$ of the total sample) who reported lifetime NSSI at baseline and completed at least one follow-up survey.

\section{Measures}

\section{Demographics}

Participants were asked to self-report age (in years), gender identity, race, ethnicity, and recent (past month) mental health treatment, which were examined as potential covariates in our analyses of interest (see below).

\section{Depressive Symptoms}


The PHQ-9 (Kroenke \& Spitzer, 2002) was used to assess symptoms of depression at baseline and at each follow-up assessment. The PHQ-9 is keyed to symptoms experienced in the past two weeks to ensure consistency with the diagnostic timeframe for a major depressive episode. Participants rate nine items on a four-point response scale ranging from 0 (not at all) to 3 (nearly every day). The PHQ-9 has recommended cut-scores for minimal (0-4), mild (5-9), moderate (10-14), moderately severe (15-19), and severe (20-27) depressive symptoms. PHQ-9 total scores at baseline showed adequate internal consistency at baseline $(\alpha=.75)$ and at each follow-up (two-week $\alpha=.82$, four-week $\alpha=.83$, six-week $\alpha=.82$ ). PHQ-9 scores were moderately correlated over time $(r s=.44-.62)$.

\section{Self-criticism}

Self-criticism was assessed using the Depressive Experiences Questionnaire SelfCriticism 6 (DEQ-SC6), a well-validated measure of self-critical feelings and beliefs (Blatt et al., 1976; Rudich et al., 2008). Participants rate six items assessing how they feel about themselves on a seven-point response scale ranging from 1 (strongly disagree) to 7 (strongly agree). The DEQ-SC6 total score showed adequate internal consistency at baseline $(\alpha=.65)$ and at followup (two-week $\alpha=.67$, four-week $\alpha=.70$, six-week $\alpha=.74$ ). DEQ-SC6 scores were moderately correlated over time $(r \mathrm{~s}=.43-.73)$.

\section{NSSI Behaviors, Characteristics, and Urges}

At baseline, participants completed the behavioral items from the Inventory of Statements About Self-Injury (ISAS; Klonsky \& Olino, 2008), which assesses 12 NSSI methods and allows participants to write an optional "other" category. Participants are asked to report the number of times in their life they have engaged in each NSSI method intentionally, and without suicidal intent. These responses are then used to create a composite measure of NSSI frequency (the sum 
of frequency responses across methods) and NSSI versatility (number of methods for which the participant endorsed any engagement). Participants were also asked to indicate the most recent time they engaged in NSSI, which was measured ordinally such that larger values indicated more recent NSSI (past week $=6$, past month $=5$, past three months $=4$, past six months $=3$, past year $=2$, prior to the past year $=1$ ). To ascertain whether participants met criterion $\mathrm{A}$ of the proposed NSSI Disorder in the fifth edition of the Diagnostic and Statistical Manual of Mental Disorders (American Psychiatric Association, 2013), participants were asked how many days they engaged in NSSI in the past year (count) and whether, at any point prior to the past year, they had engaged in NSSI at least five times within a single year period (yes or no). Baseline NSSI characteristics are available in Table 1.

At each follow-up survey, participants were asked, "over the past two weeks, how often have you had the urge or desire to engage in self-harm [i.e., intentionally injuring yourself without suicidal intent]?"). Participants responded using a seven-point rating scale ranging from never (1) to nearly all the time (7), where values of 2 or higher indicated the presence of NSSI urges. For regression analyses predicting any NSSI urges over follow-up, participants were dichotomized into those who rated this item 1 at all follow-up surveys (0) and those who rated this item 2 or higher for at least one follow-up survey (1). For analyses predicting time-varying changes in NSSI urges using path modeling, the original scale was used, specified as categorical. Participants were also asked whether they had engaged in NSSI during that two-week interval, with response options no, yes, once, and yes, more than once. Descriptive characteristics regarding follow-up NSSI urges and behaviors are provided in Table 2.

\section{Data Analytic Plans and Procedures}


First, we examined the distributions of our study variables for skewness and kurtosis. Lifetime NSSI frequency was highly skewed (9.55) and kurtotic (91.4); as a result, we chose to rank-transform this variable for subsequent analyses, with the lowest rank (1) assigned to the lowest lifetime NSSI frequency for interpretability. Next, we examined potential demographic covariates and baseline NSSI characteristics (frequency, recency, number of methods) for associations with NSSI urges reported at any follow-up assessment. Constructs that were significantly associated with follow-up NSSI urges were included as covariates in binary logistic regression models.

For hypotheses $1 \mathrm{~A}-1 \mathrm{C}$ and $2 \mathrm{~A}-2 \mathrm{C}$, binary logistic regression models were used to test the odds of any reported NSSI urges over follow-up, predicted by baseline depressive symptoms, self-criticism, and indicators of NSSI severity. Analyses were conducted in SPSS version 28, and results are presented using standardized effect sizes.

For hypotheses 3A-3B, we tested a longitudinal model using Mplus version 8.5 (Muthén \& Muthén, 2021). In this model, NSSI urges, depressive symptoms, and self-criticism were nested within individuals over time. The 7-point NSSI urge measure was specified as categorical, and all variables were regressed on themselves (auto-regressive effects) as well as on each other (cross-lagged effects), allowing for testing of lagged relationships between constructs controlling for stability in experiences over time. Further, variables were allowed to predict each other or covary within the same assessment, permitting examining of concurrent effects. Parameters were set to equivalency across follow-up intervals (e.g., the auto-regressive effect of NSSI urges from wave 2 to wave 3 was set to be equivalent to the same effect from wave 3 to wave 4). Models were calculated using maximum likelihood estimation with robust standard errors, and Monte 
Carlo integration was used to handle missing data. A correlation matrix of study variables, which includes their means and standard deviations at all time points, is provided in Table 3.

\section{Results}

\section{Descriptive Characteristics and Covariate Testing}

Participants ranged in age from $18-26(M=20.08, S D=1.80)$ years and were primarily cisgender women (78.26\%) or cisgender men (18.48\%; see Table 4 for additional demographic characteristics). Participants were primarily White $(77.17 \%)$ or Black/African-American (7.61\%), and over one-quarter of participants were Hispanic/Latinx (28.26\%).

All participants in these analyses had a lifetime history of NSSI, with over two-thirds of participants meeting the threshold for NSSI Disorder criterion A in their lifetime, and $41.76 \%$ meeting this threshold in the past year (see Table 1 for additional characteristics related to NSSI). The most reported NSSI methods were interference with wound healing (67.39\%), cutting (60.87\%), and banging/hitting oneself (53.26\%). Over half of participants $(60.87 \%)$ reported NSSI urges over follow-up, and 13.04\% reported NSSI behaviors, all of whom also reported NSSI urges (see Table 2 for follow-up NSSI characteristics).

Cisgender men and cisgender women did not differ in rates of follow-up NSSI urges $\left(\chi^{2}(1)=1.42, p=.49\right)$, nor did participants with and without recent mental health treatment at baseline $\left(\chi^{2}(1)=0.01, p=.91\right)$. However, non-Hispanic/Latinx White individuals were more likely to report follow-up NSSI urges than people from other racial/ethnic backgrounds, who were combined into a single group due to small sample sizes for specific races and ethnicities $\left(\chi^{2}(1)=4.27, p=.039\right)$. There was weak, non-significant evidence that NSSI at follow-up was associated with younger age $(t(90)=1.96, p=.05)$; however, age was not associated with PHQ-9 or DEQ-SC6 scores at baseline or follow-up (all $p s>.10$ ), and thus we elected not to control for 
age in subsequent analyses. Race/ethnicity $(0=$ non-Hispanic/Latinx White, $1=$ all other racial/ethnic groups) was included as a binary covariate in all models.

\section{Baseline NSSI Severity and Follow-Up NSSI Urges}

First, we examined whether recency of NSSI measured at baseline (modeled as an ordinal variable, reference group = past-week NSSI) would predict reporting any follow-up NSSI urges (coded $0=$ no NSSI urges over the follow-up surveys, $1=$ some degree of NSSI urges over the follow-up surveys), controlling for race/ethnicity (for full model results, see Table 5). The overall model was significant $\left(\chi^{2}(6)=18.57, p=.005, R^{2}=.20\right)$, and correctly identified $61.96 \%$ of participants with respect to follow-up NSSI. As hypothesized (1A), baseline NSSI recency was a significant predictor of reporting any follow-up NSSI urges. Specifically, participants who last engaged in NSSI more than one year ago had reduced odds of reporting any follow-up NSSI urges when compared to participants with past-week NSSI $(\mathrm{OR}=0.10, p=.007)$.

Next, we used rank-transformed NSSI frequency at baseline to predict reporting any follow-up NSSI urges, controlling for race/ethnicity. The model was significant $\left(\chi^{2}(2)=14.84, p\right.$ $\left.<.001, R^{2}=.15\right)$, correctly identifying $70.65 \%$ of participants. Baseline NSSI frequency was a significant predictor of reporting any follow-up NSSI urges, consistent with hypothesis 1B (OR $=1.03, p=.002)$.

Consistent with hypothesis $1 \mathrm{C}$, a similar pattern emerged when examining baseline NSSI versatility (number of methods) as a predictor of reporting any follow-up NSSI urges. The overall model was significant $\left(\chi^{2}(2)=13.09, p=.001, R^{2}=.13\right)$, accurately classifying $67.39 \%$ of participants. As predicted, baseline NSSI methods were associated with reporting any followup NSSI urges $(\mathrm{OR}=1.38, p=.007)$.

\section{Baseline Depression, Self-Criticism, and Follow-Up NSSI Urges}


At baseline, depressive symptoms (PHQ-9 scores) were positively correlated with indicators of NSSI severity (i.e., NSSI versatility, frequency, and recency) and with baseline selfcriticism (DEQ-SC6 scores) (see Table 3). Interestingly, baseline self-criticism was not significantly associated with indicators of NSSI versatility, frequency, or recency. As predicted, both baseline depressive symptoms (hypothesis 2A) and self-criticism (hypothesis 2B), when modeled separately, were significantly associated with reporting any follow-up NSSI urges, controlling for race/ethnicity (see Table 6 for full model results). The model examining baseline depressive symptoms as a predictor was significant $\left(\chi^{2}(2)=8.75, p=.01, \mathrm{R}^{2}=.09\right)$, correctly identifying $68.48 \%$ of participants; baseline depressive symptoms were a significant independent predictor $(\mathrm{OR}=1.13, p=.04)$. The model using baseline self-criticism to predict reporting any follow-up NSSI urges was significant $\left(\mathrm{X}^{2}(2)=11.43, p=.003, \mathrm{R}^{2}=.12\right)$, and correctly identified $66.30 \%$ of participants. Baseline self-criticism significantly predicted reporting any NSSI urges $(\mathrm{OR}=1.97, p=.01)$

In three separate models examining the predictive utility of baseline depressive symptoms jointly with indicators of baseline NSSI severity, results were consistent in showing that baseline depressive symptoms were not significantly associated with reporting any followup NSSI urges, after controlling for NSSI severity (ORs $<1.13, p s>.1$; see Table 7). This pattern was consistent with hypothesis $2 \mathrm{C}$. In contrast, and contrary to hypothesis $2 \mathrm{D}$, baseline self-criticism retained a significant association with reporting any follow-up NSSI urges in three separate models controlling for NSSI versatility, frequency, and recency (ORs $>1.80, p \mathrm{~s}<.04$; see Table 8).

\section{Concurrent Associations between Depression, Self-Criticism, and Follow-up NSSI Urges}


First, we tested separate cross-lagged models in which NSSI urges (now using the sevenpoint rating ordinal scale) and depressive symptoms or self-criticism were allowed to predict themselves and each other over time and at the same assessment wave (see Figures 1 and 2). There were significant auto-regressive effects for NSSI urges, depressive symptoms, and selfcriticism over time, such that higher levels of urges and/or symptoms at a specific follow-up predicted higher levels of the same construct at the next follow-up. Consistent with hypothesis 3A, depressive symptoms positively predicted NSSI urges at the same follow-up (OR $=1.22, p<$ $.001)$. However, contrary to hypothesis $3 \mathrm{~B}$, self-criticism did not significantly predict NSSI urges at the same follow-up $(\mathrm{OR}=1.04, p=.82)$.

When considering our exploratory aim, examining lagged, prospective associations between depressive symptoms and self-criticism and NSSI urges, the pattern of findings was reversed. Specifically, depressive symptoms did not predict NSSI urges at the following assessment $(\mathrm{OR}=0.99, p=.74)$, whereas self-criticism was significantly associated with later NSSI urges $(\mathrm{OR}=1.65, p=.01)$.

\section{Discussion}

Theoretical models of NSSI that emphasize the roles of negative affective experiences and self-perceptions highlight the potentially important roles of depressive symptoms and selfcritical cognitions in understanding NSSI urges and behaviors (e.g., Hooley \& Franklin, 2018; Klonsky et al., 2015). Prior research in this area has been limited, however, by cross-sectional designs that impede examination of the prospective, longitudinal associations of these phenomena in daily life. Further, given the relationships between depression, self-criticism, and NSSI, it remains to be seen whether these risk factors are predictive of NSSI urges when controlling for prior NSSI characteristics, which themselves are associated with subsequent NSSI 
(Fox et al., 2015). To address these gaps in the literature, we used longitudinal data from a sample of college students with elevated depressive symptoms and a lifetime history of NSSI to examine the concurrent and time-varying associations between depressive symptoms, selfcriticism, and NSSI urges over a six-week period.

Participants with greater numbers of NSSI methods and higher lifetime NSSI frequency at baseline reported NSSI urges over follow-up, consistent with prior research highlighting both of these constructs as indicators of clinical severity (Turner et al., 2013; Victor \& Klonsky, 2014). Compared to participants with NSSI in the week before enrollment, participants who had not engaged in NSSI for at least a year were less likely to report NSSI urges over follow-up. Interestingly, participants who had ceased NSSI between one week and one year ago did not differ from those with the most recent NSSI in terms of follow-up NSSI urges, suggesting that NSSI urges are likely to persist after NSSI behaviors cease (Lewis et al., 2019). This information may be particularly useful in clinical settings, insofar as it suggests the need for additional and continued support for people who have not engaged in NSSI for less than one year but have a history of NSSI behaviors.

In separate logistic regression models, both depressive symptoms and self-criticism at baseline predicted reporting any NSSI urges over the follow-up period; however, only selfcriticism remained a significant predictor of any reported later NSSI urges when controlling for indicators of prior NSSI severity. This may be due to the significant association between depressive symptoms, but not self-criticism, and NSSI severity at baseline, such that depressive symptoms have less unique predictive ability above and beyond prior NSSI history. Thus, although depression diagnosis and depressive symptoms have been prospectively associated with NSSI (Fox et al., 2015), it may be that depressive symptoms are not an effective longitudinal 
predictor of NSSI urges within young adults with elevated depressive symptoms who have already begun engaging in NSSI. This is consistent with our longitudinal path analyses, in which depressive symptoms were significantly associated with levels of concurrently assessed NSSI urges rated on a seven-point scale, but not with level of NSSI urge reported at next follow-up. In contrast, self-criticism was not significantly associated with indicators of NSSI severity at baseline, but exhibited a stronger, more robust association with experiencing any NSSI urges over follow-up, controlling for these other characteristics. In the longitudinal path analyses, this pattern was replicated. Self-criticism did not show a significant association with concurrent level of NSSI urges, but did predict increased NSSI urges at later timepoints, over and above the autoregressive effect of NSSI urges over time.

This pattern of results suggests that self-criticism may be a particularly important process to understand and address when working to ameliorate NSSI urges and behaviors among at-risk young adults. Unfortunately, self-criticism has been most frequently examined as trait-like or stable over time, resulting in relatively few studies examining within-person changes in selfcriticism over time as they relate to NSSI or other important health behaviors using ecological momentary assessment (for exceptions to this pattern, see Burke et al., 2021b and Vansteelandt et al., 2020). Further research is needed examining how self-criticism may change over shorter intervals, such as minutes or hours, how these experiences relate to both NSSI urges and behavior, and how different components of self-criticism may differentially relate to NSSI urges and behaviors, ideally using more exhaustive, multi-dimensional assessments of self-criticism.

As with any study, our methodology has notable strengths and important limitations to consider. The recruitment strategy was chosen to be more representative of college students than a sample drawn from a traditional Psychology subject pool and were screened based on elevated 
depressive symptoms, increasing the relevance of understanding NSSI in this population.

Completion rates for follow-up assessments were high, and the use of repeated measures over a six-week interval allowed for examination of longitudinal predictors of changes in NSSI risk over time, which has been rare in prior work. Further, the use of validated, appropriate measures for each study construct allows for stronger inferences based on our results. However, the sample was limited with respect to its age range (relative to the general population) and the demographics of the student body at the university where recruitment occurred; future research is needed to determine whether similar results would be found among other age groups (e.g., adolescent), young adults not enrolled in higher education, and in populations receiving more intensive psychiatric care, as well as in diverse racial and ethnic groups. Further, the endorsement of NSSI behaviors over follow-up was comparatively low relative to the endorsement of NSSI urges; thus, more work is needed to elucidate whether depression and selfcriticism contribute to the transition from NSSI urges to NSSI behaviors. Finally, although a PHQ-9 screening score of 10 or greater was required for entry into the study, baseline PHQ-9 scores were slightly lower and indicative of mild depressive symptoms $(M=8.08, S D=3.86)$, perhaps due to regression to the mean or selection biases with respect to which interested individuals ultimately attended a baseline session.

Although the incidence of NSSI behaviors over the follow-up period was too low to model in these data, NSSI urges themselves are clinically meaningful. Most individuals who engage in NSSI attempt to resist NSSI behaviors when they experience NSSI urges (Klonsky \& Glenn, 2008), and NSSI urges are associated with increased impairment and decreased quality of life among people receiving treatment for NSSI (Washburn et al., 2010). Further, NSSI urges are associated with elevated suicidal ideation (Victor et al., 2015) and prospectively predict 
increased NSSI behaviors (Turner et al., 2019), making NSSI urges critical targets for NSSI prevention and intervention efforts.

On an encouraging note, should additional research continue to support the utility of selfcriticism in predicting NSSI urges and behaviors, interventions have already been developed that appear to show efficacy in reducing self-criticism. This is particularly critical given that interventions targeting NSSI and other self-injurious behaviors, such as suicide attempts, have not improved in efficacy over the past 50 years and show consistently small effects (Fox et al., 2020). For example, a relatively brief intervention designed to increase self-worth has been shown to decrease pain tolerance and willingness to endure pain among people with a history of NSSI (Hooley \& St. Germain, 2013), and brief self-compassion exercises appear effective at creating short-term decreases in self-criticism (Kirschner et al., 2019). Compassion-focused therapy has preliminary data to support its efficacy in several clinical settings, although it has not yet been studied as an intervention for NSSI (Leaviss \& Uttley, 2015). Further, data on longerterm outcomes in self-criticism interventions are more mixed; in one study using an online daily diary intervention targeting self-criticism, effects were significant immediately post-treatment but not over a three-month follow-up, and there was no significant difference between treatment conditions in decreasing NSSI (Hooley et al., 2018). However, given that NSSI urges and behaviors typically occur over relatively brief intervals, it is possible that short-term, as-needed strategies to decrease self-criticism could be used to cope with NSSI urges and avoid NSSI behaviors among people with elevated self-criticism. 


\section{References}

American Psychiatric Association. (2013). Diagnostic and statistical manual of mental disorders: DSM-5. American Psychiatric Association.

Ben-Porath, Y. S., \& Tellegen, A. (2008/2011). Minnesota multiphasic personality inventory restructured form: Manual for administration, scoring, and interpretation. University of Minnesota Press.

Bentley, K. H., Cassiello-Robbins, C. F., Vittorio, L., Sauer-Zavala, S., \& Barlow, D. H. (2015). The association between nonsuicidal self-injury and the emotional disorders: A metaanalytic review. Clinical Psychology Review, 37, 72-88. https://doi.org/10/f68zts

Blatt, S. J., D'Afflitti, J. P., \& Quinlan, D. M. (1976). Experiences of depression in normal young adults. Journal of Abnormal Psychology, 85(4), 383-389. https://doi.org/bn5mth

Burke, T. A., Allen, K. J. D., Carpenter, R. W., Siegel, D. M., Kautz, M. M., Liu, R. T., \& Alloy, L. B. (2021a). Emotional response inhibition to self-harm stimuli interacts with momentary negative affect to predict nonsuicidal self-injury urges. Behaviour Research and Therapy, 142, 103865. https://doi.org/10/gkzqcf

Burke, T. A., Fox, K., Kautz, M. M., Rodriguez-Seijas, C., Bettis, A. H., \& Alloy, L. B. (2021b). Self-critical and self-punishment cognitions differentiate those with and without a history of nonsuicidal self-injury: An ecological momentary assessment study. Behavior Therapy, 52(3), 686-697, https://doi.org/10/ghrpw8

Burke, T. A., Piccirillo, M. L., Moore-Berg, S. L., Alloy, L. B., \& Heimberg, R. G. (2019). The stigmatization of nonsuicidal self-injury. Journal of Clinical Psychology, 75(3), 481-498. https://doi.org/10/gfzfh2 
Chapman, A. L., Gratz, K. L., \& Brown, M. Z. (2006). Solving the puzzle of deliberate selfharm: The experiential avoidance model. Behaviour Research and Therapy, 44, 371-394. https://doi.org/10/b25bt7

Claes, L., Klonsky, E. D., Muehlenkamp, J., Kuppens, P., \& Vandereycken, W. (2010). The affect-regulation function of nonsuicidal self-injury in eating-disordered patients: Which affect states are regulated? Comprehensive Psychiatry, 51, 386-392. https://doi.org/10/dx8w6w

Fox, K. R., Franklin, J. C., Ribeiro, J. D., Kleiman, E. M., Bentley, K. H., \& Nock, M. K. (2015). Meta-analysis of risk factors for nonsuicidal self-injury. Clinical Psychology Review, 42, 156-167. https://doi.org/10/f73g8s

Fox, K. R., Huang, X., Guzmán, E. M., Funsch, K. M., Cha, C. B., Ribeiro, J. D., \& Franklin, J. C. (2020). Interventions for suicide and self-injury: A meta-analysis of randomized controlled trials across nearly 50 years of research. Psychological Bulletin, 146(12), 1117-1145. https://doi.org/10/ghhd4k

Franklin, J. C., Puzia, M. E., Lee, K. M., Lee, G. E., Hanna, E. K., Spring, V. L., \& Prinstein, M. J. (2013). The nature of pain offset relief in nonsuicidal self-injury: A laboratory study. Clinical Psychological Science, 1, 110-119. https://doi.org/10/gf7skb

Franklin, J. C., Puzia, M. E., Lee, K. M., \& Prinstein, M. J. (2014). Low implicit and explicit aversion toward self-cutting stimuli longitudinally predict nonsuicidal self-injury. Journal of Abnormal Psychology, 123, 463-469. https://doi.org/10/f6mtrt

Gad, M. A., Pucker, H. E., Hein, K. E., Temes, C. M., Frankenburg, F. R., Fitzmaurice, G. M., \& Zanarini, M. C. (2019). Facets of identity disturbance reported by patients with borderline 
personality disorder and personality-disordered comparison subjects over 20 years of prospective follow-up. Psychiatry Research, 271, 76-82. https://doi.org/10/gf4htg

Gordon, K. H., Selby, E. A., Anestis, M. D., Bender, T. W., Witte, T. K., Braithwaite, S., Van Orden, K. A., Bresin, K., \& Joiner, T. E. (2010). The reinforcing properties of repeated deliberate self-harm. Archives of Suicide Research, 14(4), 329-341.

\section{https://doi.org/10/dwt3wn}

Hepp, J., Lane, S. P., Carpenter, R. W., Niedtfeld, I., Brown, W. C., \& Trull, T. J. (2017). Interpersonal problems and negative affect in Borderline Personality and Depressive Disorders in daily life. Clinical Psychological Science, 5, 470-484.

https://doi.org/10/gdcg5g

Hooley, J. M., Fox, K. R., Wang, S. B., \& Kwashie, A. N. D. (2018). Novel online daily diary interventions for nonsuicidal self-injury: A randomized controlled trial. BMC Psychiatry, 18(1). https://doi.org/10/gd6wgg

Hooley, J. M., \& Franklin, J. C. (2018). Why do people hurt themselves? A new conceptual model of nonsuicidal self-injury. Clinical Psychological Science, 6(3), 428-451. https://doi.org/10/gd3zgd

Hooley, J. M., \& St. Germain, S. A. (2014). Nonsuicidal self-injury, pain, and self-criticism: Does changing self-worth change pain endurance in people who engage in self-injury? Clinical Psychological Science, 2, 297-305. https://doi.org/10/gdcg5j

International Society for the Study of Self-Injury. (2018). What is nonsuicidal self-injury? About Self-Injury. https://itriples.org/category/about-self-injury/

Kiekens, G., Hasking, P., Claes, L., Boyes, M., Mortier, P., Auerbach, R. P., Cuijpers, P., Demyttenaere, K., Green, J. G., Kessler, R. C., Myin-Germeys, I., Nock, M. K., \& 
Bruffaerts, R. (2019). Predicting the incidence of non-suicidal self-injury in college students. European Psychiatry, 59, 44-51. https://doi.org/10/gf4htv

Kiekens, G., Hasking, P., Nock, M. K., Boyes, M., Kirtley, O., Bruffaerts, R., Myin-Germeys, I., \& Claes, L. (2020). Fluctuations in affective states and self-efficacy to resist non-suicidal self-injury as real-time predictors of non-suicidal self-injurious thoughts and behaviors. Frontiers in Psychiatry, 11, 214. https://doi.org/10/ggtqz9

Kirschner, H., Kuyken, W., Wright, K., Roberts, H., Brejcha, C., \& Karl, A. (2019). Soothing your heart and feeling connected: A new experimental paradigm to study the benefits of self-compassion. Clinical Psychological Science, 7(3), 545-565. https://doi.org/10/gfzfg3

Klonsky, E. D. (2011). Non-suicidal self-injury in United States adults: Prevalence, sociodemographics, topography and functions. Psychological Medicine, 41, 1981-1986. https://doi.org/10/b4hkkv

Klonsky, E. D., \& Glenn, C. R. (2008). Resisting urges to self-injure. Behavioural and Cognitive Psychotherapy, 36. https://doi.org/10/ddpmqf

Klonsky, E. D., Glenn, C. R., Styer, D. M., Olino, T. M., \& Washburn, J. J. (2015). The functions of nonsuicidal self-injury: Converging evidence for a two-factor structure. Child and Adolescent Psychiatry and Mental Health, 9, 44. https://doi.org/10/f8b5kp

Klonsky, E. D., \& Olino, T. M. (2008). Identifying clinically distinct subgroups of self-injurers among young adults: A latent class analysis. Journal of Consulting and Clinical Psychology, 76, 22-27. https://doi.org/10/cpnq29

Kroenke, K., \& Spitzer, R. L. (2002). The PHQ-9: A new depression diagnostic and severity measure. Psychiatric Annals, 32(9), 509-515. https://doi.org/gf6zrm 
Leaviss, J., \& Uttley, L. (2015). Psychotherapeutic benefits of compassion-focused therapy: An early systematic review. Psychological Medicine, 45(5), 927-945. https://doi.org/10/f65rj5

Lewis, S. P., Kenny, T. E., Whitfield, K., \& Gomez, J. (2019). Understanding self-injury recovery: Views from individuals with lived experience. Journal of Clinical Psychology, 75(12), 2119-2139. https://doi.org/10/gf74ng

Muthén, L. K., \& Muthén, B. O. (1998-2021). Mplus User's Guide. Eight Edition. Muthén \& Muthén.

Perkins, N. M., Ortiz, S. N., \& Smith, A. R. (2020). Self-criticism longitudinally predicts nonsuicidal self-injury in eating disorders. Eating Disorders, 28(2), 157-170. https://doi.org/10/ggf2nd

Prinstein, M. J., Heilbron, N., Guerry, J. D., Franklin, J. C., Rancourt, D., Simon, V., \& Spirito, A. (2010). Peer influence and nonsuicidal self injury: Longitudinal results in community and clinically-referred adolescent samples. Journal of Abnormal Child Psychology, 38, 669-682. https://doi.org/10/dzgpkp

Ribeiro, J. D., Franklin, J. C., Fox, K. R., Bentley, K. H., Kleiman, E. M., Chang, B. P., \& Nock, M. K. (2016). Self-injurious thoughts and behaviors as risk factors for future suicide ideation, attempts, and death: A meta-analysis of longitudinal studies. Psychological Medicine, 46, 225-236. https://doi.org/10/f84cbj

Robinson, K., \& Wilson, M. S. (2020). Open to interpretation? Inconsistent reporting of lifetime nonsuicidal self-injury across two common assessments. Psychological Assessment, 32(8), 726-738. https://doi.org/10/ggxjt9 
Rodríguez-Blanco, L., Carballo, J. J., \& Baca-García, E. (2018). Use of ecological momentary assessment (EMA) in non-suicidal self-injury (NSSI): A systematic review. Psychiatry Research, 263, 212-219. https://doi.org/10/gddm49

Rudich, Z., Lerman, S. F., Gurevich, B., Weksler, N., \& Shahar, G. (2008). Patients' selfcriticism is a stronger predictor of physician's evaluation of prognosis than pain diagnosis or severity in chronic pain patients. Journal of Pain, 9, 210-216. https://doi.org/10/c2mf3w

Taylor, P. J., Jomar, K., Dhingra, K., Forrester, R., Shahmalak, U., \& Dickson, J. M. (2018). A meta-analysis of the prevalence of different functions of non-suicidal self-injury. Journal of Affective Disorders, 227, 759-769. https://doi.org/10/gc2skk

Trepal, H. C., Wester, K. L., \& Merchant, E. (2015). A cross-sectional matched sample study of nonsuicidal self-injury among young adults: Support for interpersonal and intrapersonal factors, with implications for coping strategies. Child and Adolescent Psychiatry and Mental Health, 9, 36. https://doi.org/10/f8b68c

Turner, B. J., Baglole, J. S., Chapman, A. L., \& Gratz, K. L. (2019). Experiencing and resisting nonsuicidal self-injury thoughts and urges in everyday life. Suicide and Life-Threatening Behavior, 49(5), 1332-1346. https://doi.org/10/gfc4f6

Turner, B. J., Jin, H. M., Anestis, M. D., Dixon-Gordon, K. L., \& Gratz, K. L. (2018). Personality pathology and intentional self-harm: Cross-cutting insights from categorical and dimensional models. Current Opinion in Psychology, 21, 55-59. https://doi.org/10/gfb7kp 
Turner, B. J., Layden, B. K., Butler, S. M., \& Chapman, A. L. (2013). How often, or how many ways: Clarifying the relationship between non-suicidal self-injury and suicidality. Archives of Suicide Research, 17, 397-415. https://doi.org/10/gdcg33

Vansteelandt, K., Houben, M., Claes, L., Berens, A., Sleuwaegen, E., \& Kuppens, P. (2020). Self-criticism and dependency predict affective variability in borderline personality disorder: An ecological momentary assessment study. Personality Disorders: Theory, Research, and Treatment, 11(4), 270-279. https://doi.org/10/ggfk4q

Victor, S. E., \& Klonsky, E. D. (2014). Correlates of suicide attempts among self-injurers: A meta-analysis. Clinical Psychology Review, 34, 282-297. https://doi.org/10/f56b82

Victor, S. E., \& Klonsky, E. D. (2014). Daily emotion in non-suicidal self-injury. Journal of Clinical Psychology, 70, 364-375. https://doi.org/10/f52rvw

Victor, S. E., Scott, L. N., Stepp, S. D., \& Goldstein, T. R. (2019). I want you to want me: Interpersonal stress and affective experiences as within-person predictors of nonsuicidal self-injury and suicide urges in daily life. Suicide and Life-Threatening Behavior, 49(4), 1157-1177. https://doi.org/10/gd3z4q

Victor, S. E., Styer, D., \& Washburn, J. J. (2015). Characteristics of nonsuicidal self-injury associated with suicidal ideation: Evidence from a clinical sample of youth. Child and Adolescent Psychiatry and Mental Health, 9, 20. https://doi.org/10/f8btbp

Washburn, J. J., Juzwin, K. R., Styer, D. M., \& Aldridge, D. (2010). Measuring the urge to selfinjure: Preliminary data from a clinical sample. Psychiatry Research, 178, 540-544. https://doi.org/10/dfgv7n

Wilkinson, P., Kelvin, R., Roberts, C., Dubicka, B., \& Goodyer, I. (2011). Clinical and psychosocial predictors of suicide attempts and nonsuicidal self-injury in the Adolescent 
Depression Antidepressants and Psychotherapy Trial (ADAPT). The American Journal of Psychiatry, 168, 495-501. https://doi.org/10/ffz42f

Wolff, J. C., Thompson, E., Thomas, S. A., Nesi, J., Bettis, A. H., Ransford, B., Scopelliti, K., Frazier, E. A., \& Liu, R. T. (2019). Emotion dysregulation and non-suicidal self-injury: A systematic review and meta-analysis. European Psychiatry, 59, 25-36.

https://doi.org/10/gf26d9

Zahn, R., Lythe, K. E., Gethin, J. A., Green, S., Deakin, J. F. W., Young, A. H., \& Moll, J. (2015). The role of self-blame and worthlessness in the psychopathology of major depressive disorder. Journal of Affective Disorders, 186, 337-341. https://doi.org/f7qsdf

Zelkowitz, R. L., \& Cole, D. A. (2019). Self-criticism as a transdiagnostic process in nonsuicidal self-injury and disordered eating: Systematic review and meta-analysis. Suicide and LifeThreatening Behavior, 49(1), 310-327. https://doi.org/10/gc3gqb 
Table 1

NSSI Characteristics Reported at Baseline

\begin{tabular}{|c|c|c|}
\hline & $n(\%)$ & $M(S D)$ \\
\hline Lifetime NSSI & $92(100)$ & \\
\hline 5+ Days in Any Year with NSSI & $61(67.03)$ & \\
\hline Total Number of NSSI Episodes & & $1430(10450.94)$ \\
\hline Lifetime NSSI Methods & & $4.21(2.28)$ \\
\hline Interference with wound healing & $62(67.39)$ & \\
\hline Cutting & $56(60.87)$ & $32.78(88.39)$ \\
\hline Banging/Hitting & $49(53.26)$ & $33.73(95.28)$ \\
\hline Pinching & $43(46.74)$ & $38.17(104.68)$ \\
\hline Pulling hair & $37(40.22)$ & $21.72(70.03)$ \\
\hline Severe scratching & $39(42.39)$ & $27.14(90.79)$ \\
\hline Biting & $34(36.96)$ & $39.70(146.27)$ \\
\hline Burning & $19(20.65)$ & $1.86(7.62)$ \\
\hline Swallowing dangerous substances & $15(16.30)$ & $2.93(12.82)$ \\
\hline Rubbing with rough surfaces & $15(16.30)$ & $6.22(42.02)$ \\
\hline Sticking self with needles & $7(7.61)$ & $7.46(62.64)$ \\
\hline Other & $6(6.52)$ & $12.14(104.35)$ \\
\hline Carving & $5(5.43)$ & $0.46(2.47)$ \\
\hline \multicolumn{3}{|l|}{ Most Recent NSSI } \\
\hline Past Week & $15(16.30)$ & \\
\hline Past Month & $29(31.52)$ & \\
\hline Past 3 Months & $42(45.65)$ & \\
\hline Past 6 Months & $48(52.17)$ & \\
\hline Past 12 Months & $54(58.70)$ & \\
\hline More than 12 Months Ago & $28(30.43)$ & \\
\hline Do Not Remember & $10(10.87)$ & \\
\hline \multicolumn{3}{|l|}{ Past Year NSSI } \\
\hline 5+ Days in Past Year with NSSI & $38(41.76)$ & \\
\hline Total Number of Days with NSSI & & $20.98(47.92)$ \\
\hline
\end{tabular}

Note. NSSI = nonsuicidal self-injury. Whether or not participants had 5+ days of NSSI in any

single year was missing for $n=2$ individuals, and in the past year was missing for $n=3$

individuals. Number of days of NSSI engagement in the past year was missing for $n=2$

individuals. Most recent NSSI episode was missing for $n=1$ participant. 
Table 2

NSSI Descriptive Characteristics Reported at Each Follow-up

\begin{tabular}{lccc}
\hline & Wave 2 $(n=78)$ & Wave 3 $(n=77)$ & Wave 4 $(n=72)$ \\
& $n(\%)$ & $n(\%)$ & $n(\%)$ \\
\hline NSSI Urges & & & \\
\hline Never & $37(47.44)$ & $49(63.64)$ & $46(63.89)$ \\
Rarely & $20(25.64)$ & $15(19.48)$ & $15(20.83)$ \\
Occasionally & $12(15.38)$ & $9(11.69)$ & $6(8.33)$ \\
Sometimes & $8(10.26)$ & $2(2.60)$ & $3(4.17)$ \\
Often & $0(0)$ & $2(2.60)$ & $1(1.39)$ \\
Most of the time & $1(1.28)$ & $0(0)$ & $1(1.39)$ \\
Nearly all of the time & $0(0)$ & $0(0)$ & $0(0)$ \\
Any NSSI Urge Endorsed & $41(52.56)$ & $28(36.36)$ & $26(36.11)$ \\
\hline NSSI Behavior & & & \\
\hline No & $72(92.31)$ & $73(94.81)$ & $68(94.44)$ \\
Yes, once & $3(3.85)$ & $4(5.19)$ & $2(2.78)$ \\
Yes, more than once & $3(3.85)$ & $0(0)$ & $2(2.78)$ \\
Any NSSI Behavior Endorsed & $6(7.69)$ & $4(5.19)$ & $4(5.56)$ \\
\hline
\end{tabular}

Note. NSSI $=$ nonsuicidal self-injury. All participants who endorsed NSSI behavior at a specific assessment also endorsed non-zero NSSI urges. 


\section{Table 3}

Correlation Matrix of Study Variables

\begin{tabular}{|c|c|c|c|c|c|c|c|c|c|c|c|c|c|c|}
\hline & \multicolumn{3}{|c|}{ T1 NSSI } & \multicolumn{3}{|c|}{ Follow-Up NSSI Urges } & \multicolumn{4}{|c|}{ PHQ-9 } & \multicolumn{4}{|c|}{ DEQ-SC6 } \\
\hline & FR & MT & $\mathrm{RC}$ & $\mathrm{T} 2$ & $\mathrm{~T} 3$ & $\mathrm{~T} 4$ & T1 & $\mathrm{T} 2$ & T3 & $\mathrm{T} 4$ & T1 & $\mathrm{T} 2$ & T3 & $\mathrm{T} 4$ \\
\hline NSSI MT & $.57(92)$ & 1 & & & & & & & & & & & & \\
\hline NSSI RC & $.34(82)$ & $.33(82)$ & 1 & & & & & & & & & & & \\
\hline T2 NSSI Urges & $.24(78)$ & $.31(78)$ & $.43(71)$ & 1 & & & & & & & & & & \\
\hline T3 NSSI Urges & $.38(77)$ & $.43(77)$ & $.38(70)$ & $.47(65)$ & 1 & & & & & & & & & \\
\hline T4 NSSI Urges & $.24(72)$ & $.21(72)$ & $.30(65)$ & $.49(61)$ & $.27(60)$ & 1 & & & & & & & & \\
\hline T1 PHQ-9 & $.37(92)$ & $.23(92)$ & $.23(82)$ & .34 (78) & $.28(77)$ & $.34(72)$ & 1 & & & & & & & \\
\hline T2 PHQ-9 & $.28(78)$ & $.16(78)$ & $.14(71)$ & $.45(78)$ & $.36(65)$ & $.32(61)$ & $.45(78)$ & 1 & & & & & & \\
\hline T3 PHQ-9 & $.30(77)$ & $.17(77)$ & $.01(70)$ & $.07(65)$ & $.43(77)$ & $.09(60)$ & $.44(77)$ & $.53(65)$ & 1 & & & & & \\
\hline T4 PHQ-9 & $.38(72)$ & $.13(72)$ & $.16(65)$ & $.10(61)$ & $.27(60)$ & $.49(72)$ & $.57(72)$ & $.47(61)$ & $.62(60)$ & 1 & & & & \\
\hline T1 DEQ-SC6 & $.12(92)$ & $.20(92)$ & $.12(82)$ & $.29(78)$ & $.09(77)$ & $.20(72)$ & $.43(92)$ & $.13(78)$ & $.11(77)$ & $.30(72)$ & 1 & & & \\
\hline T2 DEQ-SC6 & $.14(78)$ & $.15(78)$ & $.06(71)$ & $.20(78)$ & $.33(65)$ & $.26(61)$ & $.22(78)$ & $.36(78)$ & $.15(65)$ & $.34(61)$ & $.56(78)$ & 1 & & \\
\hline T3 DEQ-SC6 & $.00(76)$ & $.02(76)$ & $.13(69)$ & $.08(64)$ & $.18(76)$ & $.22(59)$ & $.31(76)$ & $.34(64)$ & $.39(76)$ & $.49(59)$ & $.43(76)$ & $.53(64)$ & 1 & \\
\hline T4 DEQ-SC6 & $.26(72)$ & $.17(72)$ & $.00(65)$ & $.03(61)$ & $.16(60)$ & $.18(72)$ & $.49(72)$ & $.27(61)$ & $.26(60)$ & $.55(72)$ & $.63(72)$ & $.71(61)$ & $.73(59)$ & 1 \\
\hline $\begin{array}{l}M \\
(S D)\end{array}$ & $\begin{array}{c}1430.47 \\
(10450.94)\end{array}$ & $\begin{array}{c}4.21 \\
(2.28)\end{array}$ & $\begin{array}{c}3.29 \\
(1.97) \\
\end{array}$ & $\begin{array}{c}1.94 \\
(1.12) \\
\end{array}$ & $\begin{array}{c}1.61 \\
(0.98)\end{array}$ & $\begin{array}{c}1.63 \\
(1.05)\end{array}$ & $\begin{array}{c}8.08 \\
(3.86)\end{array}$ & $\begin{array}{c}13.55 \\
(5.10) \\
\end{array}$ & $\begin{array}{l}12.44 \\
(5.19)\end{array}$ & $\begin{array}{r}11.82 \\
(5.13) \\
\end{array}$ & $\begin{array}{c}5.37 \\
(0.91)\end{array}$ & $\begin{array}{c}5.19 \\
(0.89) \\
\end{array}$ & $\begin{array}{c}5.07 \\
(0.95)\end{array}$ & $\begin{array}{c}5.13 \\
(0.93)\end{array}$ \\
\hline
\end{tabular}

Note. Bolded values are statistically significant at $p<.05$; the sample size for each correlation is presented parenthetically.

Abbreviations: NSSI = nonsuicidal self-injury; FR = lifetime NSSI frequency (rank-transformed); MT = lifetime NSSI number of methods; RC = NSSI recency; PHQ-9 = Patient Health Questionnaire 9-item; DEQ-SC6 = Depressive Experiences Questionnaire Self-Criticism 6; T1 = time 1 (baseline data); T2 = time 2 (two-week follow-up); T3 = time 3 (four-week follow-up); T4= time 4 (sixweek follow-up). 


\section{Table 4}

Demographic and Clinical Characteristics

\begin{tabular}{lc}
\hline Variable & $n(\%)$ \\
\hline Gender Identity & $17(18.48)$ \\
\hline Cisgender male & $72(78.26)$ \\
Cisgender female & $0(0.00)$ \\
Transgender male & $0(0.00)$ \\
Transgender female & $2(2.17)$ \\
Genderqueer/gender non-conforming & $1(1.09)$ \\
Prefer not to answer & $26(28.26)$ \\
\hline Hispanic/Latinx Ethnicity & $71(77.17)$ \\
\hline Race & $7(7.61)$ \\
\hline White/Caucasian & $5(5.43)$ \\
Black/African-American & $1(1.09)$ \\
Asian/Asian-American & $0(0.00)$ \\
American Indian/Alaska Native & $8(8.70)$ \\
Native Hawaiian/Pacific Islander & $16(17.39)$ \\
Multiracial or other & $41(44.57)$ \\
\hline Baseline PHQ-9 & $32(34.78)$ \\
\hline Minimal (0-4) & $3(3.26)$ \\
Mild (5-9) & $0(0.00)$ \\
Moderate (10-14) & \\
Moderate/Severe (15-19) & \\
Severe (20-27) & \\
\hline
\end{tabular}

Note. PHQ-9 = Patient Health Questionnaire 9-item. 


\section{Table 5}

Logistic Regression Models for Indicators of NSSI Severity Predicting Follow-Up NSSI Urges

\begin{tabular}{|c|c|c|c|c|c|c|c|c|c|}
\hline & $\chi^{2}$ & $d f$ & $p$ & $R^{2}$ & $\mathrm{~B}$ & Wald & $d f$ & $p$ & $O R[95 \% \mathrm{CI}]$ \\
\hline Model 1A & 18.57 & 6 & .005 & .20 & & & & & \\
\hline Constant & & & & & 1.45 & 3.12 & 1 & .08 & 4.26 \\
\hline Race & & & & & 0.69 & 1.67 & 1 & .20 & $2.00[0.70,5.74]$ \\
\hline NSSI Recency & & & & & & 12.51 & 5 & .03 & \\
\hline NSSI Recency 1 & & & & & -2.33 & 7.22 & 1 & .007 & $0.10[0.02,0.53]$ \\
\hline NSSI Recency 2 & & & & & -0.97 & 0.68 & 1 & .41 & $0.38[0.04,3.78]$ \\
\hline NSSI Recency 3 & & & & & -1.68 & 2.19 & 1 & .14 & $0.19[0.02,1.72]$ \\
\hline NSSI Recency 4 & & & & & -1.45 & 2.25 & 1 & .13 & $0.24[0.04,1.56]$ \\
\hline NSSI Recency 5 & & & & & 0.04 & 0.001 & 1 & .97 & $1.04[0.12,8.78]$ \\
\hline Model 1B & 14.82 & 2 & $<.001$ & .15 & & & & & \\
\hline Constant & & & & & -1.35 & 6.02 & 1 & .01 & 0.26 \\
\hline Race & & & & & 1.17 & 5.81 & 1 & .02 & $3.22[1.24,8.32]$ \\
\hline NSSI Frequency & & & & & 0.03 & 9.25 & 1 & .002 & $1.03[1.01,1.05]$ \\
\hline Model 1C & 13.09 & 2 & .001 & .13 & & & & & \\
\hline Constant & & & & & -1.31 & 5.23 & 1 & .02 & 0.27 \\
\hline Race & & & & & 1.07 & 5.10 & 1 & .02 & $2.92[1.15,7.40]$ \\
\hline NSSI Versatility & & & & & 0.32 & 7.33 & 1 & .007 & $1.38[1.09,1.74]$ \\
\hline
\end{tabular}

Note. NSSI = nonsuicidal self-injury. $R^{2}$ refers to Cox \& Snell $R^{2}$. Race was dichotomized such

that $0=$ non-Hispanic/Latinx White participants, $1=$ participants of all other racial/ethnic groups.

NSSI recency was categorized with past week NSSI as the reference category, such that $1=$ more than 12 months ago, $2=6-12$ months ago, $3=3-6$ months ago, $4=1-3$ months ago, $5=1$ week to 1 month ago. NSSI frequency was rank-transformed such that larger values indicated higher lifetime frequencies. 


\section{Table 6}

Logistic Regression Models for Baseline Depression and Self-Criticism Predicting Follow-Up NSSI Urges

\begin{tabular}{lccccccccc}
\hline & $\chi^{2}$ & $d f$ & $p$ & $R^{2}$ & $\mathrm{~B}$ & Wald & $d f$ & $p$ & OR [95\% CI] \\
\hline Model 2A & 8.75 & 2 & .01 & .09 & & & & & \\
\hline Constant & & & & & -0.90 & 2.71 & 1 & .10 & 0.41 \\
Race & & & & & 0.81 & 3.14 & 1 & .08 & $2.24[0.92,5.47]$ \\
Depression & & & & & 0.13 & 4.15 & 1 & .04 & $1.13[1.01,1.28]$ \\
\hline Model 2B & 11.43 & 2 & .003 & .12 & & & & & \\
\hline Constant & & & & & -3.50 & 5.97 & 1 & .02 & 0.03 \\
Race & & & & & 0.75 & 2.59 & 1 & .11 & $2.11[0.85,5.22]$ \\
Self-Criticism & & & & & 0.68 & 6.42 & 1 & .01 & $1.97[1.17,3.33]$ \\
\hline
\end{tabular}

Note. NSSI $=$ nonsuicidal self-injury. $R^{2}$ refers to Cox $\&$ Snell $R^{2}$. Depressive symptoms were

measured using the PHQ-9 (Patient Health Questionnaire 9-item); self-criticism was measured using the DEQ-SC6 (Depressive Experiences Questionnaire Self-Criticism 6). Race was dichotomized such that $0=$ non-Hispanic/Latinx white participants, $1=$ participants of all other racial/ethnic groups. 
Table 7

Logistic Regression Models for Baseline Depressive Symptoms Predicting Follow-Up NSSI Urges, Controlling for Baseline NSSI Severity

\begin{tabular}{|c|c|c|c|c|c|c|c|c|c|}
\hline & $\chi^{2}$ & $d f$ & $p$ & $R^{2}$ & B & Wald & $d f$ & $p$ & $O R[95 \% \mathrm{CI}]$ \\
\hline Model 2C1 & 21.36 & 7 & .003 & .23 & & & & & \\
\hline Constant & & & & & 0.55 & 0.31 & 1 & .58 & 1.74 \\
\hline Race & & & & & 0.59 & 1.15 & 1 & .28 & $1.81[0.61,5.33]$ \\
\hline NSSI Recency & & & & & & 11.19 & 5 & .05 & \\
\hline NSSI Recency 1 & & & & & -2.21 & 6.38 & 1 & .01 & $0.11[0.02,0.61]$ \\
\hline NSSI Recency 2 & & & & & -0.72 & 0.36 & 1 & .55 & $0.49[0.05,5.08]$ \\
\hline NSSI Recency 3 & & & & & -1.65 & 2.07 & 1 & .15 & $0.19[0.02,1.81]$ \\
\hline NSSI Recency 4 & & & & & -1.46 & 2.20 & 1 & .14 & $0.23[0.03,1.60]$ \\
\hline NSSI Recency 5 & & & & & 0.003 & 0.00 & 1 & .99 & $1.00[0.12,8.61]$ \\
\hline Depression & & & & & 0.11 & 2.65 & 1 & .19 & $1.12[0.98,1.28]$ \\
\hline Model 2C2 & 15.69 & 3 & .001 & .16 & & & & & \\
\hline Constant & & & & & -1.66 & 6.44 & 1 & .01 & 0.19 \\
\hline Race & & & & & 1.08 & 4.82 & 1 & .03 & $2.95[1.12,7.77]$ \\
\hline NSSI Frequency & & & & & 0.03 & 6.34 & 1 & .01 & $1.03[1.01,1.05]$ \\
\hline Depression & & & & & 0.06 & 0.84 & 1 & .36 & $1.06[0.93,1.22]$ \\
\hline Model 2C3 & 15.07 & 3 & .002 & .15 & & & & & \\
\hline Constant & & & & & -1.82 & 6.90 & 1 & .01 & 0.16 \\
\hline Race & & & & & 0.96 & 3.95 & 1 & .05 & $2.60[1.01,6.69]$ \\
\hline NSSI Versatility & & & & & 0.28 & 5.47 & 1 & .02 & $1.32[1.05,1.68]$ \\
\hline Depression & & & & & 0.09 & 1.92 & 1 & .17 & $1.09[0.96,1.24]$ \\
\hline
\end{tabular}

Note. NSSI = nonsuicidal self-injury. $R^{2}$ refers to Cox \& Snell $R^{2}$. Depressive symptoms were measured using the PHQ-9 (Patient Health Questionnaire 9-item). Race was dichotomized such that $0=$ non-Hispanic/Latinx white participants, $1=$ participants of all other racial/ethnic groups. NSSI recency was categorized with past week NSSI as the reference category, such that $1=$ more than 12 months ago, $2=6-12$ months ago, $3=3-6$ months ago, $4=1-3$ months ago, $5=1$ week to 1 month ago. NSSI frequency was rank-transformed such that larger values indicated higher lifetime frequencies. 


\section{Table 8}

Logistic Regression Models for Baseline Self-Criticism Predicting Follow-Up NSSI Urges, Controlling for Baseline NSSI Severity

\begin{tabular}{|c|c|c|c|c|c|c|c|c|c|}
\hline & $\chi^{2}$ & $d f$ & $p$ & $R^{2}$ & B & Wald & $d f$ & $p$ & $O R[95 \% \mathrm{CI}]$ \\
\hline Model 2D1 & 27.65 & 7 & $<.001$ & .29 & & & & & \\
\hline Constant & & & & & -3.15 & 3.05 & 1 & .08 & 0.04 \\
\hline Race & & & & & 0.49 & 0.70 & 1 & .40 & $1.63[0.52,5.05]$ \\
\hline NSSI Recency & & & & & & 12.24 & 5 & .03 & \\
\hline NSSI Recency 1 & & & & & -2.52 & 7.32 & 1 & .01 & $0.08[0.01,0.50]$ \\
\hline NSSI Recency 2 & & & & & -0.73 & 0.37 & 1 & .55 & $0.48[0.05,5.15]$ \\
\hline NSSI Recency 3 & & & & & -1.76 & 2.26 & 1 & .13 & $0.17[0.02,1.71]$ \\
\hline NSSI Recency 4 & & & & & -1.48 & 1.96 & 1 & .16 & $0.23[0.03,1.80]$ \\
\hline NSSI Recency 5 & & & & & -0.09 & 0.01 & 1 & .94 & $0.91[0.10,8.73]$ \\
\hline Self-Criticism & & & & & 0.91 & 7.68 & 1 & .01 & $2.48[1.30,4.70]$ \\
\hline Model 2D2 & 20.44 & 3 & $<.001$ & .20 & & & & & \\
\hline Constant & & & & & -4.67 & 8.35 & 1 & .004 & 0.01 \\
\hline Race & & & & & 1.01 & 4.02 & 1 & .05 & $2.74[1.02,7.34]$ \\
\hline NSSI Frequency & & & & & 0.03 & 8.06 & 1 & .01 & $1.03[1.01,1.05]$ \\
\hline Self-Criticism & & & & & 0.65 & 5.08 & 1 & .02 & $1.91[1.09,3.35]$ \\
\hline Model 2D3 & 18.06 & 3 & $<.001$ & .18 & & & & & \\
\hline Constant & & & & & -4.27 & 7.89 & 1 & .01 & 0.01 \\
\hline Race & & & & & 0.93 & 3.61 & 1 & .06 & $2.54[0.97,6.66]$ \\
\hline NSSI Versatility & & & & & 0.29 & 5.74 & 1 & .02 & $1.34[1.05,1.70]$ \\
\hline Self-Criticism & & & & & 0.59 & 4.63 & 1 & .03 & $1.81[1.05,3.10]$ \\
\hline
\end{tabular}

Note. NSSI = nonsuicidal self-injury. $R^{2}$ refers to Cox \& Snell $R^{2}$. Self-criticism was measured

using the DEQ-SC6 (Depressive Experiences Questionnaire Self-Criticism 6). Race was dichotomized such that $0=$ non-Hispanic/Latinx white participants, $1=$ participants of all other racial/ethnic groups. NSSI recency was categorized with past week NSSI as the reference category, such that $1=$ more than 12 months ago, $2=6-12$ months ago, $3=3-6$ months ago, $4=$ 1-3 months ago, $5=1$ week to 1 month ago. NSSI frequency was rank-transformed such that larger values indicated higher lifetime frequencies. 


\section{Figure 1}

Longitudinal Model of Depression and NSSI Urges

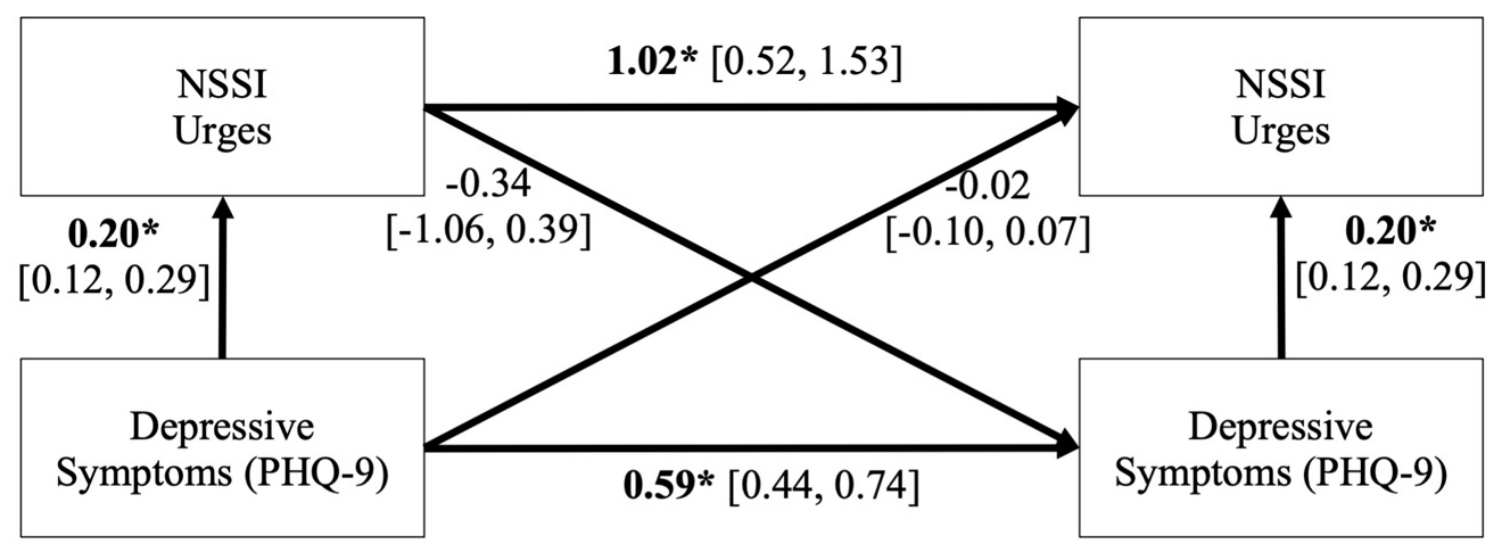

Note. NSSI = nonsuicidal self-injury. Parameters were set to equivalence across waves (e.g., the auto-regressive effect of NSSI urges from two-week to four-week follow-up was identical to the same effect from four-week to six-week follow-up. Thus, the figure demonstrates results across any two adjacent follow-up periods (two-week to four-week, four-week to six-week). Path coefficients are followed by $95 \%$ confidence intervals. Coefficients that are bolded and followed by an asterisk were statistically significant at $p<.05$. 


\section{Figure 2}

Longitudinal Model of Self-Criticism and NSSI Urges

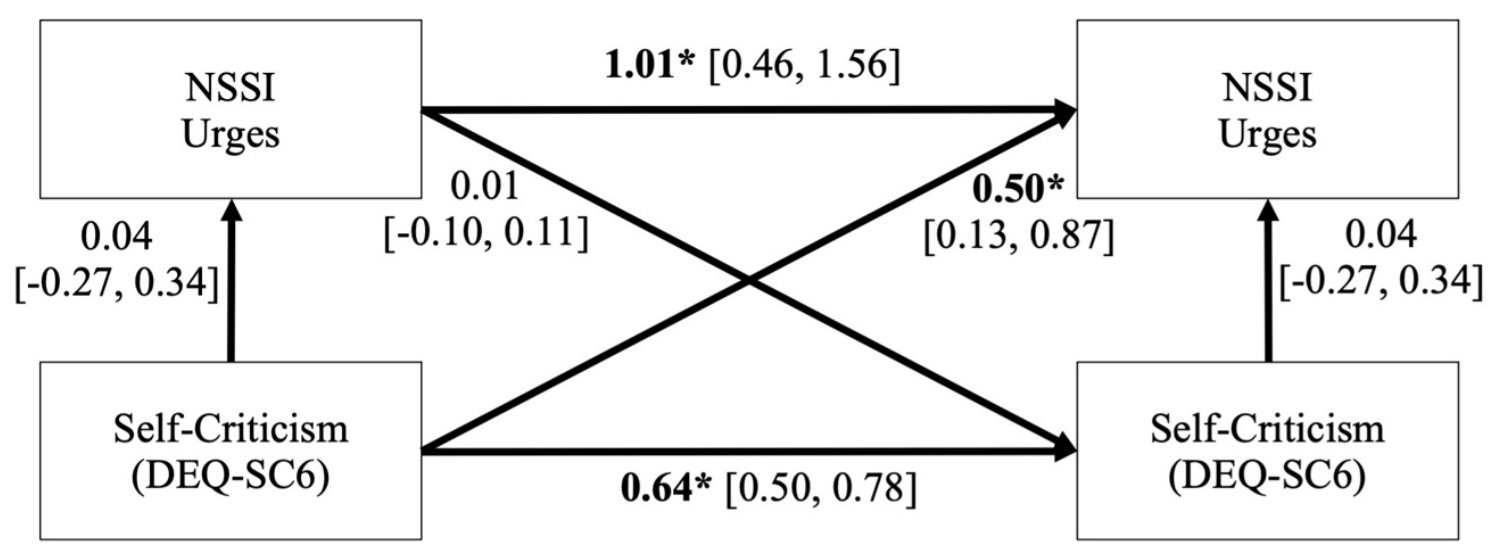

Note. NSSI = nonsuicidal self-injury. Parameters were set to equivalence across waves (e.g., the auto-regressive effect of NSSI urges from two-week to four-week follow-up was identical to the same effect from four-week to six-week follow-up. Thus, the figure demonstrates results across any two adjacent follow-up periods (two-week to four-week, four-week to six-week). Path coefficients are followed by $95 \%$ confidence intervals. Coefficients that are bolded and followed by an asterisk were statistically significant at $p<.05$. 